\title{
Effects of Aerobic Exercise on Newly Diagnosed Patients with Type 2 Diabetes in Taif, Saudi Arabia
}

\author{
Alaa A Shafie* \\ Collage of Applied Medical Sciences/High Altitude Research Centre, Taif University, Saudi Arabia \\ *Corresponding Author: Alaa A Shafie, Collage of Applied Medical Sciences/High Altitude Research Centre, Taif University, Saudi Arabia.
}

Received: July 03, 2019; Published: July 19, 2019

DOI: 10.31080/ASNH.2019.03.0373

\begin{abstract}
Type 2 diabetes (T2D) is a global burden and its prevalence increases rapidly, it is described as non-insulin dependent genetically inherited disorder, which leads to complications in patients characterized by deficiency or ineffective of the insulin released in those patients. Studies have shown than performing aerobic daily has been effective in controlling glycosylated hemoglobin A1c (HbA1c) in T2D patients. In this study, HbA1c, fasting and post-prandial blood sugar were estimated in newly diagnosed male patients 2040 year in Taif city, 40 participants were recruited and allocated equally to one of the two groups: aerobic exercise group or control group. After 8 weeks HbA1c, fasting and post-prandial blood sugar were estimated again. Our results showed significant $(\mathrm{P}<0.05)$ decrease in HbA1c in the aerobic exercise group. This is consistence with other studies that showed performing aerobic daily have positive effect on $\mathrm{HbA1c}$ in T2D patients.
\end{abstract}

Keywords: Aerobic Exercise; HbA1c; Diabetes

\section{Introduction}

Type 2 diabetes previously described as non-insulin dependent and a genetically inherited disorder, which lead to complications in patients characterized by deficiency or ineffective of the insulin released in those patients [1]. Type 2 diabetes is a global burden and it increases rapidly, prevalence of T2D is detected daily in developing and developed countries worldwide [1,2]. Diabetes is one of the oldest disease discovered by human, as Egyptians have reported diabetes long ago $[2,3]$. In additions, T2D consider to be metabolic disorder that has various effects and generally T2D patients are hyperglycemic, those patients diagnosed with T2D suffer from many complications that can lead to mortality in many of these patients due to the high prevalence rate of T2D [4,5]. Moreover, those patients are vulnerable and they suffer from atherosclerotic vascular disorders, complications in their organs such as in kidneys, eyes due to the damage in blood vessels and the nerves [2,4].
Internationally T2D inflicts a huge economic liability on healthcare providers which in turn have a great burden on countries economics [4,6]. In 2011, there were more than 360 million and this number of cases will expand to about 440 million to the year 2030 [7]. World Health Organization has ranked Saudi Arabia as the second in number of diabetes patients among middle eastern countries and seventh globally, moreover Saudi men were ranked the second after Bahrain between middle-eastern countries in number of incidence of T2D $[8,9]$. Moreover, Saudi Arabia were ranked the fourth in the world in number of type 1 diabetes globally [10]. It was reported in 1992 by Saudi ministry of health that T2D cases was less than one million, however, this number has expanded to about 2.5 million cases in 2010 [11].

Glycated hemoglobin A1c is recognized as an important indicator to detect the average blood glucose [12], it was recommended in 1976 to be used for T2D patients [13]. The standard level to iden- 
tify the individual as diabetic when HbA1c is $\geq 6.5 \%$ [12]. Glycated hemoglobin A1c is a form of hemoglobin covalently non enzymaticaly condensatted with glucose. This occurs when glucose in the blood react and bound with the exposed hemoglobin by glycation process, where glycated $\mathrm{N}$-terminal residue of hemoglobin $\beta$ chain is measured [14].

Active life and exercise are essential and are the keystones for T2D control. For patients with T2D, performing aerobic daily has been suggested, this is due to the recognized effects upon insulin sensitivity and glucose tolerance. However, not all T2D patients perform aerobic activities and achieve these suggestions [15].

According to a study performed by Liubaoerjijin., et al [16], performing exercise activity can lead to marked reduction in HbA1c in patients with T2D. Our study will be done in Taif city, the city is considered as a high-altitude with about 1800 meters above sea level, several studies have confirmed that high-altitude can have several effects on human body that are different from normal-altitude cities [17-22], therefore, our aim was to determine the influence of aerobic activities on improving glycemic control of newly diagnosed patients with T2D type 2 diabetics in Saudi people living in Taif.

\section{Material and Methods}

\section{Subjects}

Forty male subjects were recruited from King Abdel Aziz hospital, Taif, KSA. All participants have been given written informed consent to take part in the study. The study has obtained approval from research ethical committee of the Taif University. The targeted participants were as follows: recognized type 2 diabetes $<<6$ months duration), have not started injecting insulin with an inactive lifestyle, males aged between 20 to 40 years. Suitable subjects assigned when they did not show any type 1 T2D complications. Then 20 participants were allocated to aerobic exercise (AE) group or control group. The descriptive characteristics of the participants are given in Table 1.

\begin{tabular}{|l|c|c|c|c|}
\hline $\begin{array}{l}\text { Physical } \\
\text { characteristic }\end{array}$ & $\begin{array}{c}\text { Control } \\
\text { group } \\
\text { Mean } \pm \text { SD } \\
(\mathbf{n = 2 0 )}\end{array}$ & $\begin{array}{c}\text { Study } \\
\text { group } \\
\text { Mean } \pm \text { SD } \\
(\mathbf{n = 2 0 )}\end{array}$ & t -value & P-value \\
\hline Age (year) & $29 \pm 5.1$ & $28 \pm 4.1$ & 0.68 & 0.17 \\
\hline Height (cm) & $163 \pm 5$ & $164 \pm 7.2$ & 0.51 & 0.61 \\
\hline $\begin{array}{l}\text { Body weight } \\
\text { (Kg) }\end{array}$ & $59 \pm 5.6$ & $58 \pm 6.2$ & 0.53 & 0.59 \\
\hline $\begin{array}{l}\text { Body mass } \\
\text { index (Kg/m }{ }^{2} \text { ) }\end{array}$ & $22 \pm 3.2$ & $22 \pm 2.2$ & 0.00 & 0.99 \\
\hline $\begin{array}{l}\text { Average dura- } \\
\text { tion of diabe- } \\
\text { tes (month) }\end{array}$ & $5.2 \pm 0.77$ & $4.8 \pm 0.6$ & 1.83 & 0.07 \\
\hline
\end{tabular}

Table 1: Patients demographic data of control and study group.

\section{Exercise protocols}

- Aerobic exercise group (AE): Supervised aerobic exercise was carried out by the patients for 8 weeks. The exercise was performed weakly for three non-constructive days on cycle ergometers (Daum $\AA$ fitness 3, Furth, Germany). Each training session included warm up (5 $\mathrm{min})$, cycling (5 $\mathrm{min}$ ) and cool down (5 min). In order to calculate the patients' heart rate at a specific percentage of training intensity, Karvonen-Formula was used [23].

- Control group: There was no training instruction given to the subjects of the control group during the study while they were using their medications.

- Parameters: Three analytes were analyzed at baseline before the training program and at the eighth week at the end of the program. These analytes include HbA1c, fasting blood sugar (FBS) and post-prandial blood sugar (PPBS) using Nyco Card HbA1c test (NycoCard reader II, Axis Sheild PoC), glucometer (Elegance CTX-10) respectively.

- Statistical Analysis: Results are described as group mean \pm standard deviation. Paired t-test was applied to detect the differences between the groups using SPSS Software (SPSS 16.0). Statistical significance of pre- and post- training program is indicated as $\mathrm{P} \leq 0.05$ level.

\section{Results}

Forty T2D patients were evaluated in the study. After randomization, there were 20 participants in the study group and 20 in the control group. As reported in Table (1), the mean age of the control group who were not involved in the training program was $29 \pm 5.1$ year, while the mean age of the study group was of $28 \pm 4.1$ year. The mean duration of T2D (months) for control group was $5.2 \pm$ 0.77 and that for study group was $4.8 \pm 0.6$. Regarding the level of HbA1c in control group, it was $6.30 \pm 1.2$ and on the study group, it was $7.19 \pm .93$. However, the difference between control group and study group was no significant in all factors that might affect the outcome of our study such as age, height, body weight, body mass index (BMI), duration of diabetes, and the level of HbA1c.

The mean values comparison of the study and control groups for the three parameters at baseline showed that there was no significant difference between the mean values of both groups as presented in the Table 2. On the other hand, on comparison of the parameters of the two groups at 8th week, the results indicated that there was a significant difference in the mean value of $\mathrm{HbA1c}$ between the two groups $(\mathrm{P}<0.001)$. The mean value of glycosylated hemoglobin showed a mean reduction of $2.27 \%$ in the study group whereas PPBS and FBS of the two groups had no significant difference (Table 2).

\section{Discussion Discussion}

Benefits of exercise on glycaemic control are enormous [24]. Glycated hemoglobin A1c is considered a golden standard for glycemic control in diabetes as it accurately reflects glycaemic control 
over a period of time [25]. In a clinical setting, T2D usually presents with poor glycemic control, which increases the morbidity and mortality risk in the population. High HbA1c concentrations are associated with an increased risk of developing diabetic peripheral neuropathy and may also impose risks of developing diabetic retinopathy and nephropathy in due course of time [26].

\begin{tabular}{|l|c|c|c|c|c|c|}
\hline & Parameters & $\begin{array}{c}\text { Control group } \\
\text { Mean } \pm \text { SD }\end{array}$ & $\begin{array}{c}\text { Study group } \\
\text { Mean } \pm \text { SD }\end{array}$ & t -value & P-value & Sig. \\
\hline \multirow{3}{*}{ Baseline } & HbA1c & $8.02 \pm 1.47$ & $8.37 \pm 1.92$ & 0.64 & 0.52 & NS \\
\cline { 2 - 7 } & FBS & $137.17 \pm 41.14$ & $142.42 \pm 48.51$ & 0.36 & 0.71 & NS \\
\cline { 2 - 7 } & PPBS & $217.13 \pm 40.58$ & $214.82 \pm 42.73$ & 0.17 & 0.86 & NS \\
\hline \multirow{2}{*}{ At 8 weeks } & HbA1c & $8.03 \pm 1.46$ & $6.1 \pm 1.4$ & 4.26 & $<0.001$ & S \\
\cline { 2 - 7 } & FBS & $141.58 \pm 20.45$ & $131.41 \pm 23.61$ & 1.45 & 0.15 & NS \\
\cline { 2 - 7 } & PPBS & $202 \pm 33.11$ & $190 \pm 33.26$ & 1.14 & 0.26 & NS \\
\hline
\end{tabular}

Table 2: Parameters values for study and control groups before and after 8 weeks.

In the present study, aerobic exercise with standard care had an anti-glycation effect, which resulted in a mean reduction of HBA1c levels in the study group by $2.27 \%$. Increase in $\mathrm{HbA} 1 \mathrm{c}$ is associated with risks of lower extremity amputation in type 2 diabetes [27]. With every one per cent increase in HbA1c levels, there is a substantial increase in risk of lower extremity amputation [28]. A prospective study of 10 year duration found that peripheral neuropathy was an independent indicator of lower extremity amputation in type 2 diabetes [28].

There is a chance of hypoglycemia related fear as a result of the increased use of diabetes intensive therapy such as multiple insulin injections. This may affect the ability of the current medication to achieve and maintain the optimal glucose levels [27,29]. On the contrary, more prudent use of exercise with standard care as a therapy, this reduces the hypoglycemia risk, extends the period of the glycemic control, and hence reduces the risk of T2D complication.

The findings of the current research revealed that aerobic exercise of eight weeks duration even in high-altitude cities like Taif may be an imperative line of therapy for achieving glycaemic control for newly diagnosed patients with T2D.

\section{Conclusion and Limitations}

Our findings of the current research were consistent with other studies $[16,30]$ and revealed that aerobic exercise even in high-altitude cities like Taif may be an imperative line of therapy for achieving glycaemic control for newly diagnosed patients with T2D. This was clear when HbA1c has reduced significantly in T2D patients who performed aerobic exercises for eight weeks.

Our study limitations are the small study group of 40 participants only. Moreover, all participants were male and for future directions involving females is essential. All participants are from Taif city which is a high-altitude city, therefore, recruiting participants from normal-altitude cities can be more descriptive.

\section{Bibliography}

1. Berry C., et al. "Coronary Heart Disease in Patients with Diabetes. Part I: Recent Advances in Prevention and Noninvasive Management". Journal of the American College of Cardiology 49.6 (2007): 631-642.

2. Richardson SA., et al. "Growth, behaviour, and educational achievement of Jamaican children with sickle-cell trait". British Medical Journal 1.6022 (1976): 1371-1373.

3. Ahmed AM. "History of diabetes mellitus". Saudi Medical Journal 23.4 (2002): 373-378.

4. Jönsson B. "Revealing the cost of Type II diabetes in Europe". Diabetologia 45.7(2002): S5-S12.

5. Canivell S and R Gomis. "Diagnosis and classification of autoimmune diabetes mellitus". Autoimmunity Reviews 13.4-5 (2014): 403-407. 
6. Alhowaish A. "Economic costs of diabetes in Saudi Arabia". Journal of Family and Community Medicine 20.1 (2013): 1-7.

7. Chamnan P., et al. "Incidence of type 2 diabetes using proposed HbA1c diagnostic criteria in the european prospective investigation of cancer-norfolk cohort: Implications for preventive strategies". Diabetes Care 34.4 (2011): 950-956.

8. Meo SA., et al. "Prevalence of Type 2 Diabetes Mellitus Among Men in the Middle East: A Retrospective Study". American Journal of Men's Health 13.3 (2019): 155798831984857.

9. Al Dawish MA., et al. "Diabetes Mellitus in Saudi Arabia: A Review of the Recent Literature". Current Diabetes Reviews 12.4 (2016): 359-368.

10. Almehmadi M. "Interferon-stimulated-gene 15 polymorphism in Type 1 diabetic patients". Bioscience Research 16.2 (2019): 1113-1119.

11. Alotaibi A., et al. "Incidence and prevalence rates of diabetes mellitus in Saudi Arabia: An overview". Journal of Epidemiology and Global Health 7.4 (2017): 211-218.

12. Sherwani SI., et al. "Significance of HbA1c Test in Diagnosis and Prognosis of Diabetic Patients". Biomark Insights 11 (2016): 95-104.

13. Koenig RJ., et al. "Correlation of Glucose Regulation and Hemoglobin a Ic in Diabetes Mellitus". The New England Journal of Medicine 295 (1976): 417-420.

14. Miedema K. "Standardization of HbA1c and Optimal Range of Monitoring". Scandinavian Journal of Clinical Laboratory Investigation (2005): 61-72.

15. Eves ND., et al. "Resistance training and type 2 diabetes: Considerations for implementation at the population level". Diabetes Care 29.8 (2006): 1933-1941.

16. Liubaoerjijin Y., et al. "Effect of aerobic exercise intensity on glycemic control in type 2 diabetes: a meta-analysis of headto-head randomized trials". Acta Diabetol 53.5 (2016): 769781.

17. Oliver SJ., et al. "High Altitude Impairs In Vivo Immunity in Humans". High Altitude Medicine and Biology 14.2 (2013): 144149.

18. Almehmadi M. "Effect of High-Altitude on Serum-ISG-15 Levels in Influenza Patients”. 2 (2019): 2581-3226.

19. Almehmadi M., et al. "Association of high-altitude and smoking on the levels and phenotype of $\alpha \beta$-T-cells and $\gamma \delta$-T-cells". Bioscience Research 16.2 (2019): 1132-1142.
20. Alhazmi AS., et al. "Effect of High Altitude on Thyroid and Thyroid Stimulating Hormones Levels in Taif City". (2018).

21. Almehmadi M and A Shafie. "Interferon-Stimulated-Gene 15 as a Prognostic Marker of Atopic Dermatitis". ACTA Sciences Medical Sciences 3.5 (2019): 90-95.

22. Almehmadi M., et al. "Interferon-stimulated-gene-15 gene polymorphism as a risk factor in Major-Depressive-Disorder patients". Bioscience Research 15.4 (2019): 3932-3941.

23. Burgomaster KA., et al. "Divergent response of metabolite transport proteins in human skeletal muscle after sprint interval training and detraining". American journal of physiology. Regulatory, integrative and comparative physiology 292.5 (2007): R1970-R1976.

24. Little JP., et al. "A practical model of low-volume high-intensity interval training induces mitochondrial biogenesis in human skeletal muscle: potential mechanisms". The Journal of Physiology 588.6 (2010): 1011-1022.

25. Karvonen M., et al. "The effects of training heart rate: A longitudinal study". Annales Medicinae Experimentalis et Biologiae 35.3 (1957): 307-315.

26. Stratton IM., et al. "Association of glycaemia with macrovascular and microvascular complications of type 2 diabetes (UKPDS 35): prospective observational study". BMJ 321 (2000): 405-412.

27. Adler AI., et al. "Association between glycated haemoglobin and the risk of lower extremity amputation in patients with diabetes mellitus-review and meta-analysis". Diabetologia 53.5 (2010): 840-849.

28. Adler AI., et al. "Lower-extremity amputation in diabetes. The independent effects of peripheral vascular disease, sensory neuropathy, and foot ulcers". Diabetes Care 22.7 (1999): 1029 $-1035$.

29. Ismail-Beigi F., et al. "Effect of intensive treatment of hyperglycaemia on microvascular outcomes in type 2 diabetes: an analysis of the ACCORD randomised trial". Lancet 376.9739 (2010): 419-430.

30. Lee S., et al. "Exercise without weight loss is an effective strategy for obesity reduction in obese individuals with and without Type 2 diabetes". Journal of Applied Physiology 99.3 (2005): 1220-1225.

Volume 3 Issue 8 August 2019

(C) All rights are reserved by Alaa A Shafie. 